$(\mathrm{p}<0.001)$. In the interim, PrEP was widely implemented across Australia. Using interview data, we generated two themes describing condom use. Some sex workers and all of the clients we interviewed strictly refused to engage in condomless commercial sex. This strictness, however, extended only to anal sex (several participants laughed at using condoms for oral sex) and commercial encounters (many men were willing to forgo condoms for personal sex). Most male sex workers, however, were highly pragmatic about condoms. They described assessing the commercial risks-benefits of condoms, and while men were aware that condomless sex put them at risk for STIs other than HIV, they balanced that risk against increased earning potential: sex workers consistently reported charging more for condomless sex. This approach was characterized as a 'cash buffer' to compensate for the risk of being unable to work following a STI diagnosis.

Conclusion Although condom use has shifted dramatically among male sex work communities in Australia, to some they remain an important safer sex strategy. HIV PrEP and market forces have increased STI risk among male sex workers, making them ideal candidates for STI antibiotic prophylaxis.

Disclosure No significant relationships.

\section{P713 HIGH PREVALENCE OF CONDOMLESS ANAL INTERCOURSE AMONG FEMALE SEX WORKERS IN IRAN}

${ }^{1}$ Mohammad Karamouzian*, ${ }^{2}$ Azam Rahmani, ${ }^{3}$ Mostafa Shokoohi, ${ }^{3}$ Hamid Sharifi, ${ }^{4}$ Ali Mirzazadeh. ${ }^{1} B C$ Center on Substance Use, Vancouver, Canada; ${ }^{2}$ Tehran University of Medical Sciences, Tehran, Iran; ${ }^{3}$ WHO Collaborating Center for HIVISTI Surveillance, Kerman, Iran; ${ }^{4}$ UCSF, San Francisco, USA

\subsection{6/sextrans-2019-sti.777}

Background Condomless sex and particularly anal intercourse (AI) with partners and clients are among the primary drivers of the HIV epidemic among female sex workers (FSW) in Iran. However, little is known about AI prevalence and its correlates among FSW in Iran.

Methods Data were obtained from a bio-behavioral surveillance survey of 1347 FSW conducted across 13 major cities in Iran in 2015. FSW were eligible if they (i) were $\geq 18$ years of age, (ii) reported penetrative sex with more than one client in the previous year. Date were collected through one-on-one interviews using a standardized risk assessment questionnaire. A modified Poisson regression model was used to examine the correlates of recent (i.e., past-month) HAS and adjusted prevalence ratio (APR) and 95\% confidence intervals (CI) were reported (analytic sample: 1337).

Results Recent AI was reported by $18.5 \%$ (95\% CI: 13.0, 25.6) of the participants; of whom, 196 (80\%) reported inconsistent condom use during their AIs in the previous month. Recent AI was positively associated with younger $(\leq 18)$ age (APR: 1.24 ; 95\% CI: 1.06-1.59), history of group sex in the previous month (APR: $1.62 ; 95 \%$ CI: 1.08-2.44), higher number of clients in the previous month (APR: 2.35; 95\% CI: 1.38-4.00), and weekly use of alcohol (APR: 1.62; 95\% CI: 1.12-2.34). Conversely, recent AI was negatively associated with being married (APR: 0.52; 95\% CI: 0.340.80 ) and having had protected sex with their last client (APR: 0.73 ; 95\% CI: 0.54-0.97).
Conclusion About one in five FSW reported engaging in recent $\mathrm{AI}$; most of which were condomless. AI was more frequent among younger FSW and those with higher number of clients and problematic Alcohol use. Harm reduction and condom promotion efforts in Iran should include effective packages to address high prevalence of unsafe AI and re-emphasize the importance of condom use for both FSW and their partners/clients.

Disclosure No significant relationships.

\section{P714 HIV TESTING AND UNDIAGNOSED FRACTION AMONG ADOLESCENT GIRLS AND YOUNG WOMEN BY ENGAGEMENT IN SEX WORK IN MOMBASA, KENYA}

${ }^{1}$ Huiting Ma, ${ }^{2}$ Parinita Bhattacharjee, ${ }^{3}$ Linwei Wang, ${ }^{4}$ Vernon Mochache, ${ }^{5}$ Helgar Musyoki, ${ }^{6}$ Peter Gichangi, ${ }^{7}$ James Blanchard, ${ }^{8}$ Paul Sandstrom, ${ }^{8}$ Christina Daniuk, ${ }^{7}$ Stephen Moses, ${ }^{7}$ Marissa Becker*, ${ }^{9}$ Sharmistha Mishra. ${ }^{1}$ St. Michael's Hospital, Centre for Urban Health Solutions, Toronto, Canada; ${ }^{2}$ University of Manitoba, Centre for Global Public Health, Nairobi, Kenya; ${ }^{3}$ St. Michael's Hospital, Toronto, Canada; ${ }^{4}$ University of Maryland, Centre for International Health, Education and Biosecurity, Berwyn, USA; ${ }^{5}$ National AIDS and STI Control Programme, Nairobi, Kenya; ${ }^{6}$ University of Nairobi, Nairobi, Kenya; ${ }^{7}$ University of Manitoba, Centre for Global Public Health, Department of Community Health Sciences, Winnipeg, Canada; ${ }^{8}$ Public Health Agency of Canada, Winnipeg, Canada; ${ }^{9}$ St. Michael's Hospital, Li Ka Shing Knowledge Institute, Toronto, Canada

\subsection{6/sextrans-2019-sti.778}

Background Traditional HIV prevention and testing services are generally designed to either reach female sex workers or to reach adolescent girls and young women (AGYW), but not both. We sought to compare the levels and determinants of HIV testing, and the prevalence of undiagnosed HIV across subsets of AGYW in Kenya.

Methods We used data from Transitions, a 2015 cross-sectional survey of 1,299 women age 14-24 years who frequented hotspots associated with formal sex work in Mombasa, Kenya. We compared the prevalence and frequency of recent HIV testing in the previous year between AGYW involved in sex work (YSW, $\mathrm{N}=408$ ) versus those who did not sell sex (NSW, $\mathrm{N}=891$ ), and excluding those diagnosed with HIV $\geq 1$ year before the survey. We used logistic regression model to identify univariate determinants of recent testing in each group. We then compared the prevalence of undiagnosed HIV among YSW and NSW living with HIV.

Results Overall, 72\% reported a recent HIV test: $85 \%$ YSW and $65 \%$ NSW $(\mathrm{p}<0.01)$, of whom, $42 \%$ of YSW and $27 \%$ of NSW tested at least twice in the previous year $(\mathrm{p}<0.01)$. Shared determinants of recent HIV testing by subgroup included: older age (Odds Ratios [95\% Confidence Intervals] for SW: 2.8 (1.6-5.0); NSW: 2.3 [1.7-3.0]), ever contacted by peers/staff from an non-governmental/community-based organization (5.3 [1.6-32.8]; 1.9 [1.1-3.8]), prior pregnancy (1.8 [1.1-3.2]; $2.6[1.8-3.6])$, and recent STI treatment (6.2 [2.225.9]; 1.9 [1.3-3.0]). $\mathrm{N}=37 / 365$ (10\%) of $\mathrm{YSW}$ and $\mathrm{N}=30$ / 828 (4\%) NSW were living with HIV, of whom only $27 \%$ $(\mathrm{N}=10 / 37)$, and 30\% (N=9/30) were diagnosed and aware ( $\mathrm{p}=0.79$ subgroup comparison).

Conclusion Shared determinants of testing suggest that reaching across high-risk AGYW via hotspot based strategies could fill gaps left by traditional HIV prevention and testing services.

Disclosure No significant relationships. 